Revisão / Review

\title{
Tratamento da anemia ferropriva com ferro por via parenteral
}

\section{Iron deficiency anemia treatment with parenteral iron}

Rodolfo D. Cançado ${ }^{1}$

Clarisse Lobo ${ }^{2}$

João Ricardo Friedrich ${ }^{3}$

\begin{abstract}
Embora o ferro por via oral seja considerado a primeira opção de tratamento da deficiência de ferro, em algumas situações especificas, a administração de ferro por via parenteral é uma opção terapêutica que deve ser considerada. Diferentemente do ferro dextran de alto peso molecular utilizado na década de 80 e lembrado como um composto associado ao alto risco de reação anafilática e morte, o desenvolvimento e comercialização de novos compostos com ferro para uso parenteral, sobretudo por via endovenosa - como o ferro sacarato, ferro gluconato e, mais recentemente, a carboximaltose férrica -, tem se tornado cada vez mais uma alternativa terapêutica segura e efetiva, e tem possibilitado ampliar o leque de indicações desta modalidade de tratamento além da nefrologia, como obstetrícia e ginecologia, cirurgia, pediatria, gastroenterologia, hematologia e hemoterapia. Os autores revisam as principais indicações do tratamento com ferro por via parenteral, analisam as principais drogas disponiveis para a correção da anemia ferropriva por via endovenosa e propõem uma estratégia de investigação diagnóstica, tratamento e seguimento laboratorial dos pacientes com indicação desta opção terapêutica. Rev. Bras. Hematol. Hemoter. 2010; 32(Supl.2):121-128.
\end{abstract}

Palavras-chave: Deficiencia de ferro; anemia ferropriva; tratamento; compostos ferrosos; compostos férricos.

\section{Tratamento com ferro por via parenteral}

A reposição com ferro por via oral é eficaz no tratamento da maioria dos pacientes com anemia ferropriva, entretanto, em algumas situações específicas, nas quais a terapia por via oral é insuficiente para normalizar a $\mathrm{Hb}$ e/ou restabelecer os depósitos normais de ferro, a administração de ferro por via parenteral é uma alternativa eficaz, efetiva e segura, e deve ser considerada. ${ }^{1-3}$ As principais indicações de tratamento com ferro por via parenteral estão relacionadas no Quadro 1.
Diante de situações como gastroplastia redutora, gastrectomia, doença gastrintestinal inflamatória crônica, nas quais se suspeita de resposta insatisfatória com o tratamento com ferro por via oral devido à malabsorção deste elemento, um dos métodos preconizados que pode ajudar a confirmar esta alteração é o teste de absorção intestinal do ferro por via oral descrito no Quadro 2. ${ }^{2}$

Os principais medicamentos com ferro disponíveis e comercializados em diferentes países para uso por via parenteral são: ferro dextran, ferro gluconato, ferro sacarato e, mais recentemente, a carboximaltose férrica. ${ }^{4,5}$ Em função

${ }^{1}$ Médico hematologista. Professor Adjunto da Disciplina de Hematologia e Oncologia da Faculdade de Ciências Médicas da Santa Casa de São Paulo - São Paulo-SP.

${ }^{2}$ Hematologista e hemoterapeuta. Diretora do Hemocentro do Rio de Janeiro - Hemorio - Rio de Janeiro-RJ.

${ }^{3}$ Médico hematologista do Serviço de Hematologia e Transplante de Medula Óssea do Hospital de Clínicas de Porto Alegre-RS.

Faculdade de Ciências Médicas da Santa Casa de São Paulo. Setor de Gestação de Alto Risco do Departamento de Obstetrícia e Ginecologia da Irmandade da Santa Casa de Misericórdia de São Paulo - São Paulo-SP.

Correspondência: Rodolfo Delfini Cançado

Hemocentro da Santa Casa de São Paulo

Rua Marquês de Itú, $579-3^{\circ}$ andar

01223-001 - São Paulo-SP - Brasil

Tel.: (55 11) 2176-7255

E-mail:rdcan@uol.com.br

Doi: 10.1590/S1516-84842010005000066 
Quadro 1. Principais indicações do tratamento da anemia ferropriva com ferro por via parenteral'-3

- Intolerância ao ferro por via oral determinada pela ocorrência de eventos adversos que levaram ao abandono do tratamento

- Resposta insatisfatória com o ferro por via oral, geralmente devido à malabsorção, que ocorre em situações tais como: gastroplastia redutora, gastrectomia, doença gastrintestinal inflamatória crônica, incluindo infeção pelo $H$. pylori, doença celíaca e gastrite atrófica autoimune

- Situações de hemorragia recorrente (gastrointestinal, ginecológica) nas quais a quantidade de ferro absorvida por via oral não é suficiente para atender a demanda proveniente da perda excessiva de ferro secundária ao sangramento

- Anemia ferropriva intensa $(\mathrm{Hb}<8 \mathrm{~g} / \mathrm{dL})$ em paciente hemodinamicamente estável com o objetivo de se obter resposta terapêutica mais rápida e diminuir o risco da necessidade de transfusão de hemácias

- Anemia moderada a intensa em gestantes (a partir do segundo trimestre de gestação), pós-parto ou puerpério, principalmente quando há necessidade de rápida recuperação dos níveis de $\mathrm{Hb}$ e dos depósitos de ferro, minimizando eventual necessidade de transfusão de hemácias

- Resposta terapêutica mais rápida, sobretudo em pacientes em programação de cirurgia eletiva de médio a grande porte, cujo intervalo entre o tratamento e o procedimento cirúrgico seja de pelo menos 3 semanas

- Normalização mais rápida dos estoques de ferro evitando o uso prolongado da terapia por via oral e seus efeitos adversos

- Pacientes com doença renal crônica não dialítica com ferritina sérica $<100 \mathrm{ng} / \mathrm{ml}$ ou em diálise com ferritina sérica $<200 \mathrm{ng} / \mathrm{ml}$ a fim de assegurar e otimizar a resposta à administração de agente estimulador da eritropoese.

- Situações especiais, como programas de autotransfusão de pré-depósito, questões religiosas (pacientes testemunhas de Jeová)

Quadro 2. Teste de absorção intestinal do ferro por via oral

- Dosagem do ferro sérico com o paciente em jejum

- Ingestão de $60 \mathrm{mg}$ de ferro elementar por via oral com água

- Após uma a duas horas, dosar novamente o férrico sérico e comparar este valor com o do paciente em jejum

Interpretação: aumento do valor basal em jejum da concentração do ferro sérico menor do que $100 \mu \mathrm{g} / \mathrm{ml}$ sugere absorção intestinal do ferro inadequada

das propriedades farmacológicas, farmacocinéticas e de segurança dos diferentes compostos, frequência de utilização e importância a nível mundial, enfatizamos neste artigo os resultados de estudos científicos publicados e a nossa experiência com o sacarato de hidróxido férrico, que é o único produto disponível no Brasil para administração tanto por via intramuscular como por via endovenosa.

\section{Ferro dextran}

O ferro dextran é uma solução coloide de óxi-hidróxido férrico com dextran polimerizado, de peso molecular (PM) de aproximadamente $96.000 \mathrm{Da}$, que pode ser administrado por via endovenosa ou intramuscular. O ferro dextran de alto PM foi considerado, na década de 80 , como o medicamento de escolha para o tratamento da anemia ferropriva com ferro por via endovenosa.

Trata-se de uma macromolécula, de baixa estabilidade e elevada antigenicidade e, portanto, a administração tanto intramuscular como endovenosa deste composto exige a realização de dose teste com $0,5 \mathrm{~mL}$ ou $25 \mathrm{mg}$ previamente ao início do tratamento. No caso de aplicação endovenosa, dilui-se a dose total do composto a ser administrado em $500 \mathrm{~mL}$ de solução salina, administra-se como pré-medicação difeni- dramina com ou sem corticosteroide, e os primeiros $20 \mathrm{~mL}$ a $30 \mathrm{~mL}$ devem ser administrados em pelo menos cinco minutos. O paciente deve ser monitorado quanto ao aparecimento de sinais e sintomas de reações alérgica, de hipersensibilidade e anafilática. Se não houver reações adversas, após 15 a 30 minutos da dose teste, recomenda-se continuar a infusão da solução em três a quatro horas. ${ }^{1-2}$

O ferro dextran tem uma vida média plasmática de seis horas. Uma vez administrado, o ferro desliga-se do dextran e é liberado para o sistema mononuclear fagocitário, onde é armazenado sob a forma de ferritina ou transportado pela transferrina à medula óssea. A vantagem do ferro dextran é a possibilidade de se administrarem doses relativamente altas, de 500 a $2.000 \mathrm{mg}$ em uma única administração.

As principais reações locais com a aplicação do ferro dextran por via intramuscular são dor e hiperpigmentação da pele por impregnação do ferro no tecido celular subcutâneo. Com relação à aplicação endovenosa, o evento adverso (EA) mais temeroso é a reação anafilática grave, cuja incidência é da ordem de $0,5 \%$ a $1 \%$; pode ocorrer dentro de alguns minutos após o início da infusão e, em alguns casos, essa complicação é fatal. Segundo a Organização Mundial da Saúde, houve a notificação de 31 óbitos em 12 milhões de doses administradas do ferro dextran por via endovenosa, mas nenhum óbito em 25 milhões de doses administradas do ferro gluconato e ferro sacarato. ${ }^{6}$

Além disso, podem-se observar reações de hipersensibilidade tardias (de 24 a 48 horas até semanas após a aplicação) como febre, mal-estar, cefaleia, linfonodomegalia, artralgia e mialgia. A ocorrência de reação tardia é observada em mais de $10 \%$ dos pacientes tratados. 
Há alguns anos foi introduzido no mercado o ferro dextran de baixo PM, que parece tratar-se de molécula mais estável, menos imunogênica e, portanto, menor taxa de reações anafiláticas que as observadas com o ferro dextran de alto PM. Embora estudos clínicos prospectivos tenham demonstrado a eficácia e segurança deste composto em gestantes e pacientes com doença renal crônica, este produto ainda merece estudos científicos mais pormenorizados e com mais indivíduos analisados. ${ }^{1,4,6}$ Este produto não se encontra disponível no Brasil.

A constatação de EA graves mencionados anteriormente gerou, ainda na década de 80 , grande temor na utilização de ferro por via endovenosa. Por outro lado, diante da necessidade de tratamento da deficiência de ferro em grupos selecionados de pacientes, desde o início da década de 90, novos compostos com ferro para uso parenteral foram desenvolvidos e comercializados, como o ferro gluconato, o ferro sacarato e, mais recentemente, a carboximaltose férrica. Estes compostos apresentam excelente perfil de eficácia e segurança, e estão disponíveis em diversos países. ${ }^{4,6}$

\section{Ferro gluconato}

O ferro gluconato é um complexo de gluconato férrico sódico com PM aproximado de 350.000 Da, contém o mesmo núcleo de hidróxido de ferro que o ferro dextran; entretanto, utiliza-se o gluconato para estabilizar e solubilizar o composto. A ocorrência de reação anafilática grave e de reação tardia com esse complexo é da ordem de $0,04 \%$ e $0,4 \%$, respectivamente. Vários estudos têm mostrado a eficácia e segurança do ferro gluconato em pacientes em hemodiálise, no tratamento da anemia no paciente com câncer e em pacientes graves em centros de unidade intensiva. Este produto foi aprovado para comercialização nos EUA e na Europa há mais de 10 anos, porém não está disponível no território brasileiro. ${ }^{1,2,4}$

\section{Ferro sacarato}

O sacarato de hidróxido férrico ou ferro sacarato é um complexo de ferro polinuclear similar ao da ferritina (complexo proteico de ferro férrico com hidróxido de fosfato), no qual a apoferritina é substituída por um composto carbohidratado. Esta substituição é necessária pelo fato de que a ferritina possui propriedades antigênicas importantes quando administrada por via parenteral. $\mathrm{O}$ ferro sacarato contém o ferro em sua forma não iônica, como um complexo solúvel em água de ferro férrico $\left(\mathrm{Fe}^{3+}\right)$ com hidróxido de sacarato. Os filamentos polinucleares de hidróxido de ferro $\left(\mathrm{Fe}^{3+}\right)$ encontram-se rodeados perifericamente por um grande número de moléculas de sacarato unidas por ligação não covalente. ${ }^{7,8}$

Trata-se de um complexo férrico de alto PM, aproximadamente $43 \mathrm{kDa}$, que não é excretado por via renal, seguro, estável em condições fisiológicas, pois não libera ferro não iônico e com mínima imunogenicidade (ocorrência de reação alérgica $<1 / 100.000$ infusões). Estas qualidades tornaram este ferro o produto de escolha para o tratamento da anemia ferropriva em pacientes com doença renal crônica e em várias outras especialidades, como ginecologia, obstetrícia, cirurgia, gastroenterologia, hematologia e hemoterapia, na maioria dos países. ${ }^{6-8}$

No Brasil, o sacarato de hidróxido férrico é a única opção para tratamento com ferro por via parenteral. É comercializado em ampolas contendo $2 \mathrm{~mL}$ e $100 \mathrm{mg}$ de ferro elementar para uso por via intramuscular e em ampolas contendo $5 \mathrm{~mL}$ e $100 \mathrm{mg}$ de ferro elementar para uso por via endovenosa. Também está aprovado nos EUA e em vários países do continente europeu e latino-americano.

\section{Cálculo da dose e via de administração do ferro} sacarato

Para o cálculo da dose total em mg de ferro a ser reposta, pode-se utilizar a seguinte fórmula: $[\mathrm{Hb}(\mathrm{g} / \mathrm{dL})$ desejada $\mathrm{Hb}(\mathrm{g} / \mathrm{dL})$ encontrada] x peso corporal $(\mathrm{Kg}) \times 2,4+500 .{ }^{9}$

Embora a administração de ferro por via intramuscular tenha sido muito utilizada, sobretudo em função da experiência negativa com o ferro dextran por via endovenosa na década de 80 e pela facilidade de aplicação, a partir do ano 2000, esta via de administração tem sido gradativamente substituída pela via endovenosa. ${ }^{9,10}$

Recomenda-se a dose máxima diária de 200 mg (duas ampolas) de sacarato quando administrado por via intramuscular. Na prática, orienta-se a aplicação de uma ampola, uma a duas vezes por semana, até que se complete a dose total desejada ou calculada.

Sabe-se que o ferro aplicado por via intramuscular tem absorção irregular e efeitos adversos indesejáveis, como dor, mancha hipercrômica e necrose muscular (rara) no local da aplicação. A ocorrência desses EA pode ser menos frequente quando a técnica de aplicação escolhida é a intramuscular em $\mathrm{Z}$ (tema específico abordado em outro artigo deste fascículo).

As principais orientações práticas para o uso de sacarato de hidróxido férrico por via endovenosa estão relacionadas no Quadro 3.

Em crianças, recomenda-se a dose de $0,35 \mathrm{~mL} / \mathrm{kg}$ de peso corporal diluídos em, pelo menos, $200 \mathrm{~mL}$ de SF; a duração da infusão deve ser de, pelo menos, três horas; e a frequência de aplicação de apenas uma vez por semana (Bula Noripurum (Sacarato hidróxido férrico) endovenoso, 2008. Disponível em: http://www.nycomedpharma.com.br/pt/Menu/ sua_saude/Bulas/).

Estima-se que a administração de quatro ampolas (duas aplicações de $200 \mathrm{mg}$ ) do ferro sacarato seja capaz de aumentar em pelo menos $1 \mathrm{~g} / \mathrm{dL}$ a concentração de $\mathrm{Hb}$, o que corresponde ao incremento esperado da $\mathrm{Hb}$ quando se administra uma unidade de concentrado de hemácias. ${ }^{9}$

Carboximaltose férrica

Carboximaltose férrica é o composto mais recentemen- 
Quadro 3. Principais orientações práticas para o uso de sacarato de hidróxido férrico (ferro sacarato) por via endovenosa

- Não há necessidade de se realizar a dose teste de hipersensibilidade antes da aplicação

- Diluir o composto apenas em solução fisiológica (SF) a 0,9\%. Não diluir em soro glicosado

- Diluir cada ampola (5 mL, $100 \mathrm{mg}$ ) em, pelo menos, $100 \mathrm{ml}$ de SF;

- Para cada solução contendo 100 mg de ferro sacarato, o tempo de infusão deve ser de, pelo menos, 15 minutos. Portanto, a infusão da solução contendo $200 \mathrm{~mL}$ (ou mais) de SF e $200 \mathrm{mg}$ de ferro sacarato deve ser feita em, pelo menos, 30 minutos.

- É importante respeitar o tempo de infusão do medicamento

- Respeitar o intervalo entre as aplicações, que é de pelo menos 24 horas

- Respeitar o limite da dose máxima por aplicação, que é de 200 mg (2 ampolas), e da dose máxima semanal de 500 mg;

- Recomenda-se que a aplicação por via endovenosa seja feita em ambiente hospitalar ou, preferencialmente, em Serviços ou Clínicas com experiência na aplicação de medicamentos por via endovenosa por profissionais da área de enfermagem e com supervisão médica

te registrado na Europa para o tratamento da anemia ferropriva por via endovenosa que combina as propriedades positivas do ferro dextran com as do ferro sacarato.

Estudos em animais demonstraram que a dose de carboximaltose necessária para induzir toxicidade aguda é cem vezes maior comparada à dose de sulfato ferroso. Estudos clínicos em doença renal crônica, anemia pós-parto e doença inflamatória gastrointestinal demostraram tratar-se de um complexo seguro e eficaz. ${ }^{5}$

Estudo aleatório, controlado e comparativo, com o objetivo de demonstrar a não inferioridade da eficácia da carboximaltose $(n=182)$ em relação ao sulfato ferroso $(n=179)$, demonstrou que o tempo necessário para se obter a elevação da $\mathrm{Hb}$ de $2 \mathrm{~g} / \mathrm{dL}$ foi de sete dias para o grupo com carboximaltose com uma única aplicação de até $1.000 \mathrm{mg}$ e de 14 dias para o grupo com sulfato ferroso, que recebeu $325 \mathrm{mg}$ duas vezes ao dia $(p<0,001)$. O valor médio da ferritina inicial e após 42 dias de tratamento foi de $30 \mathrm{ng} / \mathrm{mL}$ e $30 \mathrm{ng} / \mathrm{mL}$ com sulfato ferroso; e de $30 \mathrm{ng} / \mathrm{mL}$ e $230 \mathrm{ng} / \mathrm{mL}$ com carboximaltose $(\mathrm{p}<0,05) .{ }^{11}$

A vantagem mais importante deste produto em relação ao ferro sacarato é que a carboximaltose pode ser administrada em dose de até $1.000 \mathrm{mg}$ de ferro infundida em apenas 15 minutos, com risco mínimo de reação adversa. Esta posologia facilita o tratamento, evita perda de tempo e a necessidade do paciente ter de retornar ao serviço várias vezes durante a terapia.

Este produto encontra-se em processo de aprovação pelas autoridades regulatórias de saúde dos EUA e de vários países latino-americanos, inclusive do Brasil.

\section{Perfil de segurança dos produtos com ferro}

administrados por via endovenosa

Distúrbios gastrointestinais - náusea, vômito, dor abdominal, diarreia ou obstipação - são frequentemente observados em pacientes tratados com ferro por via oral e raramente em pacientes que recebem ferro por via endovenosa, exceto pelo gosto metálico relatado por porcentagem significativa dos pacientes tratados por esta via.

Em 2008, Ganguli et al. ${ }^{6}$ publicaram estudo prospectivo, randomizado, comparando o perfil de segurança entre ferro dextran de baixo PM, ferro gluconato e ferro sacarato no tratamento de pacientes com doença renal crônica. Foram realizadas 2.980 infusões de ferro por via endovenosa em 339 pacientes e, em $49(14,45 \%)$ deles, documentou-se o total de 56 eventos adversos ( $1,88 \%$ por infusão). O ferro sacarato foi o complexo mais seguro, com menor incidência de EA e menor taxa de descontinuidade do tratamento.

O Quadro 4 compara os principais produtos com ferro disponíveis para uso endovenoso.

Quadro 4. Comparação entre os principais compostos com ferro para uso endovenoso $0^{2,6}$

\begin{tabular}{|c|c|c|c|}
\hline Parâmetro & $\begin{array}{l}\text { Ferro dextran } \\
\text { de alto PM }\end{array}$ & $\begin{array}{c}\text { Ferro } \\
\text { sacarato }\end{array}$ & $\begin{array}{l}\text { Carboximaltose } \\
\text { férrica }\end{array}$ \\
\hline $\begin{array}{l}\text { Dose máxima em } \\
\text { mg por aplicação }\end{array}$ & $500-1000$ & 200 & $500-1000$ \\
\hline $\begin{array}{l}\text { Necessidade de } \\
\text { dose teste }\end{array}$ & Sim & Não & Não \\
\hline $\begin{array}{l}\text { Tempo de } \\
\text { infusão }\end{array}$ & 2 a $4 h$ & $\begin{array}{l}100 \mathrm{mg} \text { em } \\
15 \text { minutos }\end{array}$ & $\begin{array}{l}500-1000 \mathrm{mg} \\
\text { em } 15 \text { minutos }\end{array}$ \\
\hline Reação anafilática & Incomum & Muito rara & Muito rara \\
\hline Reação tardia & Comum & Incomum & Incomum \\
\hline
\end{tabular}

\section{Anemia, gestação, cirurgia e tratamento com ferro endovenoso}

Vários estudos clínicos, particularmente conduzidos pela equipe do Dr. Breymann, ${ }^{12}$ têm demonstrado não apenas a eficácia e segurança do uso do ferro sacarato no tratamento de gestantes com anemia ferropriva e da anemia pós-parto, mas a melhora das condições gerais da paciente e redução da necessidade transfusional. Estes fatos também têm 
sido constatados no tratamento de pacientes com doenças inflamatórias gastroinstestinais, particularmente conduzidos pelo Dr. Gashe e colaboradores. ${ }^{13}$

Cançado et al. ${ }^{9}$ avaliaram a eficácia e segurança do uso do ferro sacarato por via endovenosa (200 mg, uma vez por semana) em cinquenta pacientes com anemia ferropriva intolerantes ou refratários ao ferro por via oral e observaram aumento médio do valor de $\mathrm{Hb}$ de $3,61 \mathrm{~g} / \mathrm{dL}$ para as mulheres e de 4,83 g/dL para os homens com a administração de um número médio de 12 ampolas por paciente, demonstrando um aumento significativo da $\mathrm{Hb}$ [valor médio inicial e final de $8,48 \mathrm{~g} / \mathrm{dLe} 12,34 \mathrm{~g} / \mathrm{dL}$, respectivamente $(\mathrm{p}<0,001)]$, e da ferritina sérica [valor médio inicial e final de 4,65 g/dL e 93,20 g/dL; respectivamente $(\mathrm{p}<0,0001)]$ após seis semanas de tratamento. Se fossem administradas duas doses semanais de ferro sacarato, o incremento de $\mathrm{Hb}$ observado poderia ter ocorrido em apenas três semanas, o que comprova a eficácia e importância deste composto no tratamento da anemia. Neste estudo, nenhum paciente recebeu transfusão de hemácias.

A busca de alternativas à transfusão de hemácias em procedimentos cirúrgicos programados ou eletivos tem sido objeto de estudo de vários grupos de pesquisadores, ${ }^{14-19}$ que têm demonstrado a importância da avaliação, condução e correção pré-operatória da anemia como um dos principais fatores favoráveis em relação ao sucesso do procedimento cirúrgico e em relação ao paciente propriamente dito, uma vez que a correção da anemia melhora as condições gerais do paciente, diminui os riscos de complicações peri-operatórias, reduz a necessidade de transfusão de hemácias alogênicas e o tempo de internação. Estes resultados podem ser ainda melhores quando se associa eritropoetina recombinante ao ferro endovenoso.

Recente meta-análise, ao comparar o uso de ferro sacarato endovenoso $(n=181)$ e grupo controle sem ferro endovenoso $(\mathrm{n}=200)$ em pacientes submetidos à prótese de quadril ou joelho, mostrou que o grupo tratado com ferro

Tabela 1. Taxa de transfusão de hemácias, infecção e mortalidade em pacientes submetidos à prótese de quadril tratados ou não com ferro sacarato endovenoso

\begin{tabular}{ccccc}
\hline Parâmetro & $\mathrm{N}$ & $\begin{array}{c}\text { Transfusão } \\
\text { de hemácias } \\
\mathrm{n}(\%)\end{array}$ & $\begin{array}{c}\text { Infecção } \\
\mathrm{n}(\%)\end{array}$ & $\begin{array}{c}\text { Mortalidade } \\
30 \text { dias } \mathrm{n}(\%)\end{array}$ \\
\hline $\begin{array}{c}\text { Grupo } \\
\text { Controle } \\
\text { (sem ferro } \\
\text { endovenoso }\end{array}$ & 200 & $107(53,5)$ & $68(34)$ & $34(17,0)$ \\
$\begin{array}{c}\text { Grupo com } \\
\text { ferro sacarato } \\
\text { endovenoso }\end{array}$ & 181 & $56(30,9)$ & $27(14,9)$ & $14(7,7)$ \\
$\begin{array}{c}\text { RR [IC95\%] } \\
(p)\end{array}$ & 381 & $0,58[0,45-0,74]$ & $0,44[0,29-0,65]$ & $0,45[0,25-0,82]$ \\
$(p<0,001)$ & $(p<0,001)$ & $(p<0,007)$ \\
\hline
\end{tabular}

$\mathrm{RR}=$ risco relativo; $\mathrm{IC}=$ intervalo de confiança endovenoso apresentou taxas significativamente menores de transfusão de hemácias, infecção e mortalidade nos primeiros trinta dias de cirurgia, conforme pode-se observar na Tabela 1.

Na última década, o número de indivíduos submetidos à gastroplastia redutora tem aumentado exponencialmente; entretanto, o seguimento clínico e a preocupação com as complicações pós-operatórias destes pacientes, sobretudo da deficiência de ferro, tem sido muito aquém do esperado ou desejado. ${ }^{20}$

A frequência de pacientes que desenvolvem deficiência de ferro nos dois primeiros anos após a cirurgia bariátrica varia entre $15 \%$ e $60 \%$, e as principais causas desta complicação são: intolerância às carnes em geral, diminuição da secreção do ácido gástrico e exclusão do duodeno do trânsito alimentar, que corresponde à principal área de absorção do ferro proveniente da dieta. ${ }^{20,21}$ Além disso, mulheres em idade fértil, gestantes e adolescentes requerem atenção especial, pois apresentam maior predisposição à anemia ferropriva. ${ }^{20,21}$

O perfil de segurança e as propriedades farmacocinética e farmacodinâmica conferem e asseguram ao ferro sacarato administrado por via endovenosa excelente resposta terapêutica e tornam este composto uma opção de grande valia.

Entretanto, vale a pena lembrar e destacar que o alto grau de tecnologia necessário para a produção de compostos como este leva-nos a duvidar ou, pelo menos, a questionar, quanto a bioequivalência, perfil de eficácia e segurança de compostos diferentes do original comercializados por diferentes fabricantes em diferentes países, conforme estudo clínico experimental. ${ }^{22}$

\section{Estratégia de investigação diagnóstica, tratamento e seguimento laboratorial do paciente com anemia ferropriva em programação cirúrgica}

O Quadro 5 relaciona os principais exames laboratoriais, diagnóstico de anemia ferropriva e diagnóstico diferencial das anemias hipocrômicas microcíticas necessários para a correta abordagem do paciente com anemia e em programação cirúrgica.

A perda de $500 \mathrm{ml}$ de sangue corresponde à perda de cerca de $200 \mathrm{mg}$ de ferro. A administração de $200 \mathrm{mg}$ de ferro sacarato por via endovenosa é capaz de promover um aumento médio da $\mathrm{Hb}$ de $0,5 \mathrm{~g} / \mathrm{dL}$ (podendo variar entre $0,25 \mathrm{~g}$ a $1 \mathrm{~g} / \mathrm{dL}$ ). Portanto, para se obter um incremento do valor da $\mathrm{Hb}$ de $1 \mathrm{~g} / \mathrm{dL}$ são necessários 400 $\mathrm{mg}$ de ferro sacarato endovenoso (duas doses de $200 \mathrm{mg}$ ) e, via de regra, esse é o aumento obtido com a administração de uma unidade de concentrado de hemácias, que contém cerca de $200 \mathrm{mg}$ de ferro. 
Quadro 5. Abordagem laboratorial pré-operatória para o diagnóstico da anemia ferropriva e diagnóstico diferencial das anemias hipocrômicas e microcíticas

\section{Exames laboratoriais} necessários

Diagnóstico de anemia

Diagnóstico de anemia ferropriva

Principais doenças que devem ser investigadas no diagnóstico diferencial da

Anemia Hipocrômica Microcítica

Hemograma completo, contagem de reticulócitos, dosagem de ferro sérico, capacidade total de ligação de ferro e ferritina, proteína $\mathrm{C}$ reativa, eletroforese de hemoglobina

Pacientes com $\mathrm{Hb}<13 \mathrm{~g} / \mathrm{dl}$ (homens), $<12 \mathrm{~g} / \mathrm{dL}$ (mulheres), $<11 \mathrm{~g} / \mathrm{dL}$ (gestantes)

Anemia hipocrômica microcítica e saturação da transferrina* $<20 \%$ e ferritina $<15 \mathrm{ng} / \mathrm{mL}$ para crianças e $<30 \mathrm{ng} / \mathrm{mL}$ para adultos

Anemia hipocrômica microcítica ou normocrômica normocítica e saturação da transferrina $<20 \%$ e Ferritina $>100 \mathrm{ng} / \mathrm{mL}$ sugerem o diagnóstico de anemia de doença crônica ou anemia da inflamação. Neste caso, contagem de reticulócitos, dosagem da creatinina sérica, proteína $\mathrm{C}$ reativa $\mathrm{e}$, eventualmente, da eritropoetina sérica, podem auxiliar na confirmação do diagnóstico. Tratamento com ferro endovenoso associado ou não à eritropoetina recombinante pode ser considerado

Anemia hipocrômica microcítica ou normocrômica normocítica e saturação da transferrina $<20 \%$ e ferritina entre 30 e $100 \mathrm{ng} / \mathrm{mL}$ sugerem a associação de anemia por deficiência de ferro e inflamação. Nestes casos, a contagem de reticulócitos, proteína C reativa e receptor solúvel da transferrina (RsTr) ou a relação entre RsTr e log da ferritina, grau de hipocromia ou concentração da $\mathrm{Hb}$ reticulocitária podem auxiliar na confirmação do diagnóstico. Tratamento com eritropoetina recombinante pode ser considerado

Contagem normal ou aumentada de glóbulos vermelhos, RDW menor que $18 \%$, predomínio de microcitose, redução discreta da hemoglobina $\mathrm{A}$ e aumento da hemoglobina $\mathrm{A}_{2}$ sugerem fortemente o diagnóstico de talassemia-beta menor. A presença de resistência globular aumentada é exame diagnóstico auxiliar. Entretanto, em alguns casos, apenas a análise molecular do DNA pode fornecer o diagnóstico definitivo. Por outro lado, a associação entre talassemia- $ß$ menor e anemia ferropriva pode ocorrer, reduzindo a concentração de hemoglobina $A_{2}$. Logo, para evitar falsos diagnósticos, na suspeita desta associação deve-se corrigir o estoque de ferro corpóreo para avaliação posterior da eletroforese de hemoglobina.

*Saturação da transferrina (\%) = Ferro/capacidade total de ligação de ferro x 100; RDW= red distribution width

Quadro 6. Orientações para uso de ferro sacarato por via endovenosa em pacientes em programação de cirurgia eletiva (de médio ou grande porte) ou gestantes com anemia ferropriva

Grau de Anemia
(Valor de Hemoglobina)

Anemia leve $(\mathrm{Hb}$ entre $10 \mathrm{~g} / \mathrm{dL}$ e abaixo do limite inferior de normalidade, ou seja, $<13 \mathrm{~g} / \mathrm{dL}$ para homens, $<12 \mathrm{~g} / \mathrm{dL}$ para mulheres e $<11 \mathrm{~g} / \mathrm{dL}$ para gestantes

Anemia moderada* ( $\mathrm{Hb}$ entre 8 e $10 \mathrm{~g} / \mathrm{dL}$ ) a grave ${ }^{* *}(\mathrm{Hb}<8 \mathrm{~g} / \mathrm{dL})$
Administrar $200 \mathrm{mg}$ de ferro sacarato, $2 \times /$ semana, nas duas semanas que antecedem o procedimento (exemplo: dia - 14 e - 10, e dia - 7 e -3) e no dia da cirurgia ou parto

\section{A recomendação inicial é administrar} $200 \mathrm{mg}$ de ferro sacarato, 1x/semana, nas duas semanas que antecedem $o$ procedimento (exemplo: dia - 14, dia - 7) e no dia da cirurgia ou parto

e em cond
Administração adicional de $200 \mathrm{mg}$ de ferro sacarato para cada $500 \mathrm{ml}$ de sangue "perdido" no peri-operatório. Pode-se administrar uma única dose de $200 \mathrm{mg}$ (exemplo: dia +2 ou +3 ) para aqueles que tiveram perda sanguínea de até $500 \mathrm{~mL}$ ou administrar duas doses de $200 \mathrm{mg}$ (exemplo: dia +2 ou +3 e no dia +5 ou +6 ) para aqueles com perda sanguínea entre 500 e $1000 \mathrm{~mL}$ no peri-operatório. Pode-se repetir o esquema na semana seguinte para aqueles com perda sanguínea $>1000 \mathrm{~mL}$
Tratamento
Pós-operatório 
uma dose um dia antes da cirurgia ou parto, e uma dose após. $\mathrm{O}$ tratamento pós-operatório segue as mesmas recomendações descritas para pacientes anêmicos dependendo da quantidade da perda sanguínea (Quadro 6).

Se em torno do sétimo dia pós-cirurgia o paciente ainda estiver com ferritina sérica menor que $30 \mathrm{ng} / \mathrm{mL}$ ou anemia ferropriva, recomenda-se administrar uma ou mais doses de $200 \mathrm{mg}$ de ferro sacarato, uma vez por semana, até a obtenção de ferritina igual ou maior que $30 \mathrm{ng} / \mathrm{mL}$.

Para pacientes submetidos a gastroplastia redutora, preconiza-se o seguimento trimestral no primeiro ano após a cirurgia, semestral no segundo e terceiro anos; e anual por tempo indefinido. Quanto aos exames laboratoriais para avaliação da quantidade de ferro do organismo, recomenda-se, para cada consulta, a realização de hemograma completo, contagem de reticulócitos, dosagem de ferro sérico, capacidade total de ligação de ferro, ferritina sérica, proteína $\mathrm{C}$ reativa. A dosagem sérica da vitamina $B_{12}$ e do folato pode se solicitada a cada seis meses.

\section{Conclusões}

- A reposição com ferro por via oral é eficaz no tratamento da maioria dos pacientes com anemia ferropênica, entretanto, em algumas situações específicas, nas quais a terapia por via oral é insuficiente para normalizar a $\mathrm{Hb}$ e/ou restabelecer os depósitos normais de ferro, a administração de ferro por via parenteral deve ser considerada.

- O desenvolvimento e comercialização de compostos com ferro para uso parenteral, sobretudo por via endovenosa, tem se tornado cada vez mais uma alternativa terapêutica segura e efetiva e possibilitado ampliar o leque de indicações desta modalidade de tratamento, além da nefrologia como obstetrícia e ginecologia, cirurgia, pediatria, gastroenterologia, hematologia e hemoterapia.

- A investigação e correção da anemia no pré-operatório parece ser um dos principais fatores favoráveis em relação ao sucesso do procedimento cirúrgico e em relação ao paciente propriamente dito, uma vez que a correção da anemia melhora as condições gerais do paciente, diminui os riscos de complicações peri-operatórias, reduz a necessidade de transfusão de hemácias alogênicas e o tempo de internação. Estes resultados podem ser ainda melhores quando se associa eritropoetina recombinante ao ferro endovenoso

- A abordagem pré-operatória do paciente com anemia deve incluir, pelo menos, a realização de hemograma completo, contagem de reticulócitos, dosagem de ferro sérico, capacidade total de ligação de ferro e ferritina sérica, para a confirmação da deficiência de ferro

- O seguimento clínico, monitoração do status de ferro e estratégia terapêutica adequado dos pacientes submetidos à gastroplastia redutora são fundamentais e devem ser realizados regularmente e por tempo indefinido
- É fundamental o empenho do médico clínico ou cirurgião, em conjunto com o hematologista e hemoterapeuta, com o objetivo de melhorar as condições clínicas do paciente, oferecendo-lhe opções terapêuticas de menor risco de agravo à sua saúde, e isto requer seguimento médico e laboratorial periódico e adequada estratégia de tratamento.

\section{Abstract}

Although oral iron is generally considered the first choice in the treatment of iron deficiency, in some specific situations, parenteral iron administration is a therapeutic option that should be considered. Different to the high-molecular-weight iron dextran utilized in the eighties and remembered as a compound associated with a high risk of anaphylaxis and death, the development and marketing of newer preparations for parenteral, in particular endovenous, administration, such as iron sucrose, ferric gluconate and more recently ferric carboxymaltose, are becoming a more effective and safe therapeutic alternative, that have extended the range of indications beyond nephrology to obstetrics and gynecology, surgery, pediatrics, gastroenterology, hematology and hemotherapy. The authors review the main indications of parenteral iron treatment, analyse the drugs available for the correction of iron deficiency anemia by intravenous iron administration and propose a new strategy of diagnostic investigation, treatment and laboratory follow up of the patient with indication for this therapeutic option. Rev. Bras. Hematol. Hemoter. 2010;32(Supl.2):121-128.

Key words: Iron deficiency; anemia, iron-deficiency; therapeutics; ferrous compounds; ferric compounds.

\section{Referências Bibliográficas}

1. Baynes RD. Iron deficiency. In: Brock JH, Halliday JW, Pippard MJ, Powell LW. Iron metabolism in health disease. London, W.B. Saunders, 1994. p.189-225.

2. Cook JD. Diagnosis and management of iron-deficiency anemia. Best Pract Res Clin Haematol. 2005;18(2):319-32.

3. Beutler E. Disorders of iron metabolism. In: Williams Hematology. Chapter 40. Seventh Edition. McGraw-Hill; 2006; 511-553.

4. Beris P, Maniatis A. Role of intravenous iron therapy in anemia management: state of the art. Semin Hematol 2006; 43(6):S1-S2.

5. Lyseng-Williamson KA, Keating G. Ferric carboxymaltose. A review of its use in iron-deficiency anaemia. Drugs. 2009;69 (6):739-56.

6. Anirban G, Kohli HS, Jha V, Gupta KL, Sakhuja V. The comparative safety of various intravenous iron preparations in chronic kidney disease patients. Ren Fail. 2008;30(6):629-38.

7. Geisser P. Safety and efficacy of iron(III)-hydroxide polymaltose complex. A review of over 25 years experience. Arzneimittelforschung. 2007;57(6A):439-52.

8. Toblli JE, Brignolli R. Iron(III)-hydroxide polymaltose complex in iron deficiency anemia / review and meta-analysis. Arzneimittelforschung. 2007;57(6A):431-8.

9. Cançado RD et al. Avaliação da eficácia do uso intravenoso de sacarato de hidróxido de ferro III no tratamento de pacientes adultos com anemia ferropriva. Rev Bras Hematol Hemoter. 2007; 29:147-53. 
10. Awerbach M, Goodnough LT, Picard D, Maniatis A. The role of intravenous iron in anemia management and transfusion avoidance. Transfusion. 2008;48(5):988-1000.

11. Van Wyck DB, Martens MG, Seid MH, Baker JB, Mangione A. Intravenous ferric carboxymaltose compared with oral iron in the treatment of postpartum anemia: a randomized controlled trial. Obstet Gynecol. 2007;110(2 Pt 1):267-78.

12. Breymann C. The use of iron sucrose complex for anemia in pregnancy and the post partum period. Hematology. 2006;43 (6):S28-S31.

13. Gashe C, Kulnigg S. Intravenous iron in inflammatory bowel disease. Hematology. 2006;43(6):S18-S22.

14. Cuenca J, García-Erce JA, Martínez AA, Solano VM, Molina J, Muñoz M. Role of parenteral iron in the management of anaemia in the elderly patient undergoing displaced subcapital hip fracture repair: preliminary data. Arch Orthop Trauma Surg. 2005;125 (5):342-7.

15. Cuenca J, García-Erce JA, Martínez F, Pérez-Serrano L, Herrera A, Muñoz M. Perioperative intravenous iron, with or without erythropoietin, plus restrictive transfusion protocol reduce the need for allogeneic blood after knee replacement surgery. Transfusion. 2006;46(7):1112-9.

16. Cuenca J, García-Erce JA, Muñoz M. Efficacy of intravenous iron sucrose administration for correcting preoperative anemia in patients scheduled for major orthopedic surgery. Anesthesiology. 2008;109(1):151-2

17. García-Erce JA, Cuenca J, Muñoz M, Izuel M, Martínez AA, Herrera A, Solano VM, Martínez F. Perioperative stimulation of erythropoiesis with intravenous iron and erythropoietin reduces transfusion requirements in patients with hip fracture. A prospective observational study. Vox Sang, 2005;88(4):235-43.

18. García-Erce JA, Cuenca J, Haman-Alcober S, Martínez AA, Herrera A, Muñoz M. Efficacy of preoperative recombinant human erythropoietin administration for reducing transfusion requirements in patients undergoing surgery for hip fracture repair. An observational cohort study. Vox Sang. 2009;97(3):260-7.

19. Muñoz M, García-Erce JA, Cuenca J. Management of postoperative anemia after lower limb arthroplasty in patients over 65 years old. Anesth Analg. 2009;108(3):1045-6.

20. Love AL, Billett HH. Obesity, bariatric surgery, and iron deficiency: true, true, true and related. Am J Hematol. 2008;83(5):403-9.

21. Varma S, Baz W, Badine E, Nakhl F, McMullen H, Nicastro J et al. Need for parenteral iron therapy after bariatric surgery. Surg Obs Relat Dis. 2008;4(6):715-9.

22. Toblli JE, Cao G, Oliveri L, Angerosa M. Differences between original intravenous iron sucrose and iron sucrose similar preparations. Arzneimittelforschung. 2009;59(4):176-90.

O tema foi sugerido e avaliado pelo coeditor deste fascículo educativo, Rodolfo Delfini Cançado, e pelo board interno da RBHH, e publicado após a concordância do editor, Milton Artur Ruiz.

Conflito de interesse: sem conflito de interesse

Recebido: 08/01/2010

Aceito: $01 / 02 / 2010$ 